\title{
Dieta y disponibilidad de forraje del venado cola blanca Odocoileus virginianus thomasi (Artiodactyla: Cervidae) en un campo experimental de Campeche, México
}

\author{
Danilo Granados ${ }^{1}$, Luis Tarango ${ }^{2}$, Genaro Olmos ${ }^{2 *}$, Jorge Palacio ${ }^{2}$, Fernando Clemente $^{2}$ \& \\ Germán Mendoza ${ }^{3}$ \\ 1. Programa de Ganadería, Colegio de Postgraduados, Montecillo, México; granados.lorenzo@colpos.mx \\ 2. Programa de Manejo y Conservación de Fauna Silvestre, Colegio de Postgraduados, San Luis Potosí, México; \\ ltarango@colpos.mx,olmosg@colpos.mx,.jpalacio@colpos.mx, clemente@colpos.mx \\ 3. Departamento de Producción Agrícola y Animal, Universidad Autónoma Metropolitana, Xochimilco, México; \\ gmendoza@correo.xoc.uam.mx \\ * Correspondencia.
}

Recibido 22-VII-2013. Corregido 15-XII-2013. Aceptado 15-I-2014.

\begin{abstract}
Forage use and availability for white tailed deer Odocoileus virginianus thomasi (Artiodactyla: Cervidae) in an experimental unit of Campeche, Mexico. In Campeche state, 122 Wildlife Conservation and Management Units have been recently conformed. In these units, eventhough the white tailed deer Odocoileus virginianus thomasi is a game species, no studies on its diet have been undertaken. The objectives of this work were to estimate the botanical composition of the diet and its seasonal change, to determine forage availability, carrying capacity and stocking rate of $O$. virginianus thomasi. The study was conducted in the experimental unit of Colegio de Postgraduados in Campeche, Mexico, from October 2010 to May 2012. The diet was determined through microhistological analyses of the white tailed deer feces by the use of reference material. Forage availability was determined through the Adelaide's method; the stocking rate, using the grazing pressure factor; and carrying capacity considering forage availability and $35 \%$ of utilization efficiency. In this experimental unit, the deer diet included 40 species belonging to 15 families. The highest species richness ocurred during the rainy season with 29 species. However, deers preferred shrubs during all seasons, and herbaceous species during the rainy season. The diet composition, forage availability, carrying capacity and stocking rate varied throughout the year. Carrying capacity ranged from 0.04 to $1.08 \mathrm{deer} / \mathrm{ha}$. Additional studies are required to detail about the composition of the diet, habitat availability and use throughout its geographical range, and to detail on nutritional and health aspects. Rev. Biol. Trop. 62 (2): 699-710. Epub 2014 June 01.
\end{abstract}

Key words: carrying capacity, stocking rate, seasonal variation, tropical habitat, food habits, microhistological analysis.

En el estado de Campeche, México durante 1999 al 2010, se conformaron 122 Unidades de Manejo para la Conservación de la Vida Silvestre o UMAS (SEMARNAT, 2011) con una superficie de 761690 ha (13\% del territorio Campechano). En ellas se aprovecha el venado cola blanca, y es una de las especies más estudiadas en el norte, centro y sur de México (Mandujano, 2004; Plata et al., 2009). Sin embargo, las subespecies tropicales han sido poco investigadas (Weber, García \& ReynaHurtado, 2006), particularmente $O$. virginianus thomasi (Mandujano, 2004), que se distribuye desde el sur de Veracruz y Oaxaca hasta Tabasco, Chiapas y en la región costera de Campeche (Halls, 1984; Galindo \& Weber, 1998).

Durante su alimentación, los animales herbívoros seleccionan las plantas que les proporcionan el beneficio energético más alto (Cordova, Wallace \& Pieper, 1978); en este 
proceso, obtienen el alimento del ambiente, lo digieren, absorben y metabolizan (Forbes, 1988; Robbins, 2001). Específicamente, los ungulados como el venado cola blanca, seleccionan plantas para su mantenimiento, producción, y para enfrentar condiciones climáticas severas y depredación (Provenza, 1995; Bailey et al., 1996). La disponibilidad de forraje se refiere a las especies vegetales presentes en una determinada superficie y accesibles para el animal (Allison, 1985), esta disponibilidad no solo varía con la época del año, condiciones climáticas, fertilidad del suelo y carga animal, sino que determina el cómo los animales hacen uso del forraje en el transcurso del año (Allison, 1985; National Research Council, 1987; Distel, Villalba \& Laborde, 1994; Enríquez, Bolaños \& Meléndez, 1999). Dependiendo de las condiciones del hábitat, las especies consumidas puede ser 53 como en el estado de Morelos (Vásquez, 2009), 81 (Ramírez, 2004), 109 (Arceo, 2003) o hasta 139 especies (Villareal-Espino et al., 2011) en la Mixteca Poblana. En general, los estudios indican que los arbustos y las herbáceas son los componentes más frecuentes comparados con el consumo de pastos y especies arbóreas (DiMare, 1994; Arceo, Mandujano \& Gallina, 2005).

La capacidad de carga $(\mathrm{K})$ desde un enfoque de uso y disponibilidad de hábitat, se refiere al número de individuos que pueden ser sostenidos sin que exista un deterioro del hábitat (Gallina, 1993), y desde el punto de vista demográfico, $\mathrm{K}$ se refiere a una población en equilibrio donde su crecimiento se estabiliza cuando las tasas de natalidad y mortalidad se igualan (Akçakaya, Burgman \& Ginzburg, 1999). De manera general, K indica el número promedio de individuos que un hábitat puede albergar durante un año, sin que estos individuos ni los recursos naturales se dañen (Holechek, Pieper \& Herbel, 1995). Otro concepto relacionado con la capacidad de carga es el de carga animal (CA), el cual, a diferencia de K, expresa la variación estacional en el número de individuos que un hábitat en específico puede sostener (Holechek et al., 1995; Holechek et al., 2003).
Para conservar y manejar adecuadamente a O. virginianus thomasi y su hábitat en México, se requiere conocer el uso del hábitat, específicamente, la composición de su dieta, la disponibilidad y consumo del forraje, y la capacidad de carga en áreas tropicales. Por lo anterior, los objetivos de este trabajo fueron determinar la composición botánica de la dieta y la disponibilidad del forraje por época del año, así como la capacidad de carga y carga animal del $O$. virginianus thomasi en el estado de Campeche, México, que contribuyera con una base informativa para su manejo en la zona.

\section{MATERIALES Y MÉTODOS}

Área de estudio: El estudio se realizó en el Campo Experimental del Colegio de Postgraduados, Campus Campeche, de octubre 2010 a mayo 2012. Este Campo Experimental se ubica en el km 17.5 de la Carretera Federal Haltuchén-Edzná en el Municipio de Champotón, Estado de Campeche, México, y tiene una extensión de 82ha; las coordenadas geográficas son $\left(19^{\circ} 29^{\prime} 56.80^{\prime \prime} \mathrm{N}-90^{\circ} 32^{\prime} 34.65^{\prime \prime} \mathrm{W}\right.$; $19^{\circ} 29^{\prime} 46.02^{\prime \prime} \mathrm{N}-90^{\circ} 32^{\prime} 21.89^{\prime \prime} \mathrm{W} ; 1^{\circ} 29^{\prime}$ 48.01 " N - 90 31' 56.64" W; 19०30' 11.56 " $\mathrm{N}-90^{\circ} 32$ ' $13.55^{\prime} \mathrm{W}$ ).

Según la clasificación de Köppen, modificada por García, (1981), el clima predominante en el área de estudio es $\mathrm{AW}_{1}$ (Cálido Húmedo), la precipitación anual varía entre 900 y 1 $200 \mathrm{~mm}$; de esta cantidad, aproximadamente el $85 \%$ se presenta entre mayo y octubre, el resto de noviembre a abril. La temperatura media anual fluctúa de 25.5 a $26.4^{\circ} \mathrm{C}$, la máxima de 33.8 a $36.6^{\circ} \mathrm{C}$ durante mayo y junio, y la mínima de 15.1 a $19.4^{\circ} \mathrm{C}$ en enero García (1981). Los suelos son litosoles de textura media, y el tipo de vegetación que predomina es la selva mediana subperenifolia (Cuanalo, Ojeda, Santos \& Ortiz, 1989).

Trabajo de campo y análisis de laboratorio: Para determinar la composición de la dieta del venado cola blanca, se recolectó muestras de las plantas disponibles en el área de estudio durante 2010. Las plantas se identificaron y se 
utilizaron para elaborar el catálogo de referencias microhistológicas (Peña \& Habib, 1980). Asimismo, durante 2011-2012, y mediante recorridos aleatorios de campo (Lemos, Rojas \& Zuñiga, 2005) dentro del área de estudio, se recolectó muestras de grupos fecales frescos por época del año (lluvia-agosto 2011; transición-enero 2012 y seca-mayo 2012), las cuales se identificaron con la fecha, época del año, coordenadas geográficas, se secaron a $65^{\circ} \mathrm{C}$, trituraron y almacenaron hasta su análisis. El tamaño de muestra se estimó según Anthony \& Smith (1974); y para los análisis del material de referencia y de las muestras de grupos fecales, se siguió la técnica microhistológica (Holechek, 1982).

Para el análisis de la dieta se formaron cinco muestras mixtas por época del año (100g de heces de cada grupo fecal por época), de cada muestra mixta se observaron 10 campos (objetivo 10x y ocular 10x) dando un total de 50 campos por época del año. Las plantas consumidas se identificaron comparando las estructuras epidérmicas (tamaño, forma y disposición de las células, estomas, tricomas, glándulas, cristales, paredes celulares, células de silicio) con las del catálogo de referencia (Peña \& Habib, 1980).

La disponibilidad de forraje se evaluó en seis parcelas permanentes de $20 \times 20 \mathrm{~m}$, estas se seleccionaron al azar a partir de un mapa del área de estudio con cuadrícula de 50x50m, en donde cada uno de los vértices de los cuadros constituyó una parcela con posibilidad de ser seleccionada. La disponibilidad del forraje se determinó por especie, estrato vegetativo (arbustivo, herbáceo, pasto y arbóreo) y por época del año; la disponibilidad de arbustivas y arbóreas se estimó con el método de Adelaide (Andrew, Noble \& Lange, 1979), y la de hierbas y pastos, se cuantificó dentro de seis parcelas permanentes en tres subparcelas de un metro cuadrado cada una (18 subparcelas en total), cortando el material vegetal a ras de suelo. Durante el muestreo, se excluyeron los pastos muertos y altamente lignificados, los cuales no son apetecibles para el venado (Martínez, Molina, González, Marroquín \&
Navar, 1997; Janis, Damuth \& Theodor, 2000; Clemente et al., 2005). Las muestras vegetales fueron clasificadas, secadas a $65^{\circ} \mathrm{C}$ y pesadas, con la información de los pesos del material seco, se calculó la biomasa disponible por especie, estrato vegetativo y época del año.

La $\mathrm{K}$ se estimó con el modelo descrito por Holechek et al. (1995). La CA se estimó con el modelo basado en la presión de pastoreo descrito por Paladines \& Lazcano (1983). Para ambas estimaciones, se utilizó la disponibilidad de materia seca (MS) $\mathrm{kgMS} / \mathrm{ha}$ de los diferentes estratos vegetativos (arbustivo, herbáceo, pasto y arbóreo) y la MS total. Para ello, se consideró una eficiencia de utilización del forraje del 35\% (Gotelli, 2008), la superficie total del campo experimental (82ha) y un consumo de MS de $3.5 \%$ del peso vivo de un venado de $45 \mathrm{~kg}$ (Stuth \& Sheffield, 2001). La $\mathrm{K}$ se estimó considerando los 365 días del año y para la CA 93, 123 y 149 días para lluvia, transición y seca, respectivamente.

Durante el análisis de la dieta, se obtuvo la frecuencia de aparición de plantas identificadas por género y especie; con esta información, se calculó la densidad relativa por medio del cuadro de Fracker \& Brichle (1944). La riqueza y diversidad de especies en la dieta por época del año se determinó con el índice de ShannonWiener (H') log base 10, ambos atributos se analizaron con pruebas de t-Student modificada por Hutcheson (Zar, 1999).

Para analizar la disponibilidad de forraje de especies arbóreas y arbustivas (seis repeticiones), herbáceas y pastos (nueve repeticiones) por época del año, se aplicó un análisis de varianza y las medias se compararon con la prueba de Tukey $(\mathrm{p}<0.05)$ del paquete SAS (SAS Institute, 2008); para identificar diferencias en el consumo de especies vegetales por estrato vegetativo y época del año, se aplicó la prueba de heterogeneidad de ji cuadrada (Zar, 1999).

La similitud de la composición botánica de la dieta a nivel de especie y entre épocas del año, se determinó con el índice de Kulczynski (Hubálek, 1982) y la asociación entre la época del año y el consumo de plantas por estrato 
CUADRO 1

Composición botánica de la dieta del venado cola blanca (Odocoileus virginianus thomasi) en un campo experimental de Campeche, México

TABLE 1

Diet composition of white tailed deer (Odocoileus virginianus thomasi) in an experimental unit of Campeche, México

\begin{tabular}{|c|c|c|c|c|c|}
\hline \multirow{2}{*}{ Estrato vegetativo } & \multirow{2}{*}{ Familia } & \multirow{2}{*}{ Especie } & \multicolumn{3}{|c|}{ Porcentaje de aparición por época } \\
\hline & & & Lluvia & Transición & Seca \\
\hline \multirow[t]{4}{*}{ Arbóreo } & Euphorbiaceae & Gymnanthes lucida & 0.4 & 3.8 & 0.0 \\
\hline & Compositae & Lasianthaea fruticosa & 0.7 & 0.0 & 0.0 \\
\hline & Malvaceae & Heliocarpus appendiculatus & 0.0 & 0.0 & 4.3 \\
\hline & Fabaceae & Tamarindus indica & 0.4 & 0.0 & 0.0 \\
\hline \multirow[t]{12}{*}{ Arbustivo } & Caesalpiniaceae & Senna pallida & 4.9 & 2.1 & 0.0 \\
\hline & Euphorbiaceae & Manihot glaziovii & 1.5 & 2.8 & 2.8 \\
\hline & Euphorbiaceae & Croton peraeruginosus & 2.3 & 0.0 & 0.0 \\
\hline & Fabaceae & Caesalpinia pulcherrima & 1.5 & 4.2 & 4.9 \\
\hline & Fabaceae & Acacia glomerosa & 3.6 & 2.4 & 0.0 \\
\hline & Fabaceae & Bahuinia divaricata & 10.4 & 13.5 & 21.2 \\
\hline & Fabaceae & Acacia gaumeri & 1.9 & 0.0 & 7.8 \\
\hline & Fabaceae & Mimosa bahamensis & 0.0 & 3.8 & 0.0 \\
\hline & Fabaceae & Leucaena leucocephala & 0.0 & 8.1 & 13.5 \\
\hline & Malvaceae & Sida glabra & 1.1 & 4.6 & 0.0 \\
\hline & Myrtaceae & Eugenia $\mathrm{sp.}$ & 11.1 & 10.2 & 0.0 \\
\hline & Rutaceae & Citrus limon & 0.7 & 3.1 & 0.0 \\
\hline \multirow[t]{24}{*}{ Herbáceo } & Asclepiadaceae & Asclepia curassavica & 1.1 & 0.0 & 0.0 \\
\hline & Asclepiadaceae & Cynanchum racemosum & 0.0 & 2.4 & 0.0 \\
\hline & Asteraceae & Conyza sp. & 1.1 & 0.0 & 0.0 \\
\hline & Caesalpiniaceae & Senna sp. & 1.1 & 0.6 & 0.0 \\
\hline & Compositae & Verbesina encelioides & 1.5 & 0.0 & 0.0 \\
\hline & Compositae & Isocarpha oppositifolia & 9.2 & 11.5 & 3.3 \\
\hline & Convolvulaceae & Ipomoea $\mathrm{sp}$. & 0.7 & 1.2 & 0.0 \\
\hline & Compositae & Verbesina encelioides & 1.5 & 0.0 & 0.0 \\
\hline & Compositae & Isocarpha oppositifolia & 9.2 & 11.5 & 3.3 \\
\hline & Convolvulaceae & Ipomoea $\mathrm{sp}$. & 0.7 & 1.2 & 0.0 \\
\hline & Convolvulaceae & Ipomoea anisomeres & 5.9 & 4.6 & 0.0 \\
\hline & Convolvulaceae & Ipomoea purpurea & 3.1 & 0.0 & 0.0 \\
\hline & Convolvulaceae & Merremia aegyptia & 0.0 & 1.5 & 0.0 \\
\hline & Fabaceae & Mucana pruriens & 2.7 & 0.0 & 0.0 \\
\hline & Fabaceae & Centrosema virginianum & 2.3 & 0.0 & 0.0 \\
\hline & Lamiaceae & Salvia curviflora & 0.0 & 0.6 & 0.0 \\
\hline & Malvaceae & Hampea trilobata & 9.2 & 5.0 & 0.0 \\
\hline & Malvaceae & Hibiscus spiralis & 2.3 & 0.0 & 2.3 \\
\hline & Malvaceae & Abutilon sp. & 0.7 & 0.0 & 0.0 \\
\hline & Sapindaceae & Serjania meridionalis & 1.9 & 0.0 & 0.0 \\
\hline & Acanthaceae & & 0.0 & 0.9 & 0.0 \\
\hline & Convolvulaceae & & 2.3 & 0.0 & 0.0 \\
\hline & Malvaceae & & 1.5 & 0.0 & 0.0 \\
\hline & Verbenaceae & & 0.0 & 0.0 & 1.4 \\
\hline \multirow[t]{3}{*}{ Pasto } & Poaceae & Saccharum officinarum & 0.0 & 4.2 & 5.4 \\
\hline & Poaceae & Paspalum blodgettil & 0.0 & 0.0 & 2.3 \\
\hline & Poaceae & Sporobolus indicus & 0.0 & 0.0 & 0.9 \\
\hline \multicolumn{3}{|c|}{ Fragmentos no identificables } & 13.0 & 9.0 & 30.0 \\
\hline \multicolumn{3}{|l|}{ Total } & 100 & 100 & 100 \\
\hline
\end{tabular}


vegetativo, se determinó utilizando un análisis de correspondencia (SAS Institute, 2008). Asimismo, para determinar si algún estrato vegetativo fue seleccionado o evitado por el venado cola blanca, considerando la cantidad de sus componentes (biomasa $\mathrm{kgMS} / \mathrm{ha}$ ) y su uso (frecuencia en la dieta), se corrió un análisis de uso-disponibilidad (Neu, Beyers \& Peek, 1974; Byers, Steinhorst \& Krausman, 1984).

\section{RESULTADOS}

Durante el estudio se recolectaron 21 muestras de grupos fecales, cantidad suficiente para la determinación precisa de la dieta del venado cola blanca. La dieta del venado cola blanca en el área de estudio incluyó 40 especies de 15 familias (Cuadro 1), de las cuales las familias: Fabaceae, Malvaceae y Convolvulaceae fueron las más frecuentes con nueve, cinco y cuatro especies, respectivamente. Las arbustivas (48\%) y herbáceas (27.2\%) fueron las más consumidas por el venado, mientras que los pastos $(4.2 \%)$ y especies arbóreas $(3.2 \%)$ lo fueron en menor proporción.

La riqueza de la dieta por época fue de 29 (lluvia), 21 (transición) y 12 especies (seca). La diversidad fue mayor en la época de lluvia que en la seca $(\mathrm{p}=0.001)$ y que en la de transición $(\mathrm{p}=0.002)$, la riqueza de la de transición fue mayor que la de la época seca $(\mathrm{p}=0.001)$.

CUADRO 2

Disponibilidad de forraje para el venado cola blanca en un campo experimental de Campeche, México

TABLE 2

Forage availability for white tailed deer in an experimental unit of Campeche, Mexico

\begin{tabular}{lccc}
\multirow{2}{*}{ Estrato vegetativo } & \multicolumn{3}{c}{ Época del año kgMS/ha } \\
\cline { 2 - 4 } & Lluvia & Transición & Seca \\
Pasto & $275.0^{\mathrm{a}}$ & $60.3^{\mathrm{b}}$ & $27.2^{\mathrm{b}}$ \\
Herbáceo & $214.2^{\mathrm{a}}$ & $108.0^{\mathrm{b}}$ & $54.0^{2 \mathrm{ab}}$ \\
Arbustivo & $286.7^{\mathrm{a}}$ & $129.5^{\mathrm{b}}$ & $99.5^{\mathrm{b}}$ \\
Arbóreo & $23.8^{\mathrm{a}}$ & $10.4^{\mathrm{b}}$ & $9.5^{\mathrm{b}}$ \\
\hline
\end{tabular}

ab Medias con distinta literal en el mismo renglón son diferentes $(\mathrm{p}<0.05)$.
La equitatividad fue de $E=0.9116$ en la de lluvia, $\mathrm{E}=0.9167$ para transición, y $\mathrm{E}=0.9316$ en seca, mientras que el índice de similitud de la composición botánica de la dieta fue de 56.4 (lluvia vs. transición), 36.9 (seca vs. transición) y $24.4 \%$ (lluvia vs. seca).

La disponibilidad de forraje fue mayor en la época de lluvia en todos los estratos vegetativos; sin embargo, las arbustivas contribuyeron con mayor biomasa en el año (Cuadro 2) y las arbóreas con menor cantidad. El mayor componente de la dieta fueron las herbáceas en la época de lluvia, las arbustivas en transición, las arbustivas y pastos en la época seca, y las arbóreas y pastos las menos frecuentes durante el año (Cuadro 3).

El análisis de correspondencia identificó una asociación entre la época de lluvia y el estrato herbáceo; asimismo, las arbustivas y arbóreas se asociaron a la época de transición, mientras que los pastos y arbóreas a la época seca (Fig. 1). El venado cola blanca utilizó de manera diferente los cuatro estratos vegetativos, seleccionó las arbustivas en las tres épocas del año, evitó los pastos en la época de lluvia y transición, y las hierbas en la época de seca; la forma arbórea se utilizó de acuerdo a su disponibilidad durante el año (Cuadro 4).

La K varió de 0.04 a 1.08 venados/ha estimados con la MS de las arbóreas y total, respectivamente; por su parte la CA fluctuó

CUADRO 3

Frecuencia de plantas en la dieta del venado cola blanca por estrato vegetativo y época del año

TABLE 3

Plant frequency in the diet of white tailed deer per vegetative strata and season of the year

\begin{tabular}{lcccc}
\multirow{2}{*}{ Estrato vegetativo } & \multicolumn{3}{c}{ Época del año } & Total \\
& Lluvia & Transición & Seca & \\
Herbáceo & 113 & 81 & 15 & 209 \\
Arbustivo & 97 & 152 & 85 & 334 \\
Arbóreo & 4 & 12 & 9 & 25 \\
Pasto & 0 & 13 & 18 & 31 \\
No identificable & 33 & 30 & 59 & 122 \\
Total & & & & 721 \\
& \multicolumn{4}{l}{$\mathrm{X}^{2=117.5, \text { g.1. }=8,(\mathrm{p}<0.0001)}$} \\
\hline
\end{tabular}




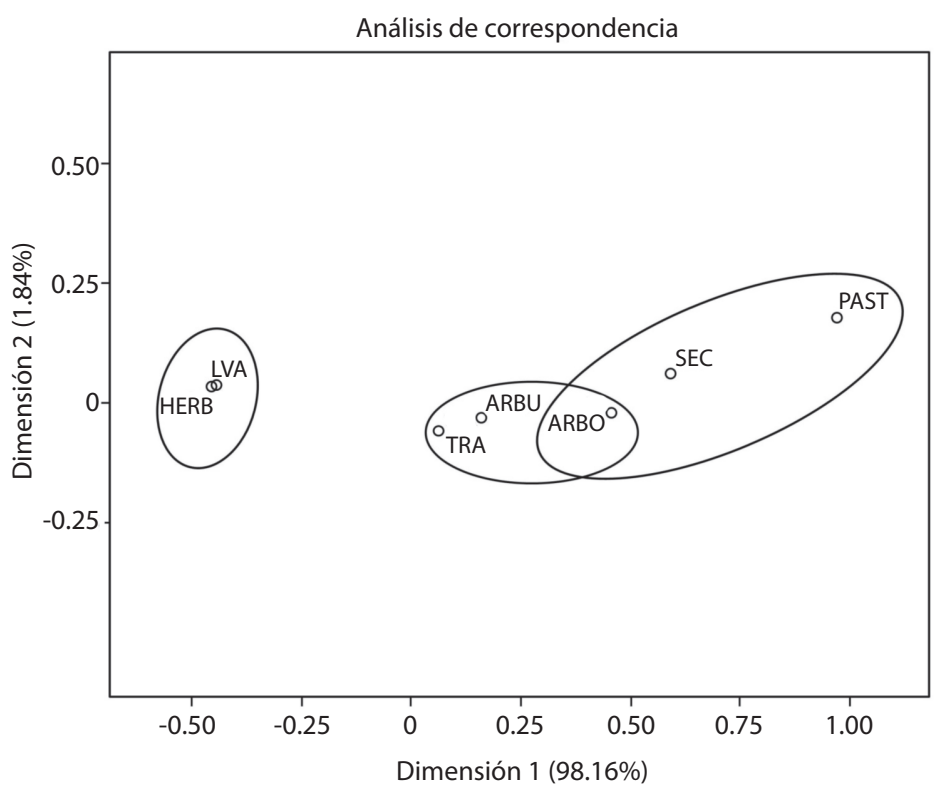

Fig. 1. Análisis de correspondencia entre estratos vegetativos y épocas del año. LVA=época de lluvia, TRA=época de transición, $\mathrm{SEC}=$ época seca; $\mathrm{HERB}=$ herbáceo, $\mathrm{ARBU}=$ arbustivo, $\mathrm{ARBO}=$ arbóreo, $\mathrm{PAST}=$ pasto.

Fig. 1. Correspondence analysis among vegetative strata and seasons of the year. $L V A=$ rainy season, $T R A=$ transition season, $\mathrm{SEC}=$ dry season; $\mathrm{HERB}=$ herbaceous, $\mathrm{ARBU}=$ shrub, $\mathrm{ARBO}=$ tree, $\mathrm{PAST}=$ grass.

de 0.02 con la MS de las arbóreas en la época seca a 1.14 venados/ha con la de las arbustivas en la época de lluvia, en este estudio el forraje de las especies arbóreas fue el forraje limitante, el cual resultó en los valores más bajos de $\mathrm{K}$ y CA (Cuadro 5).

\section{DISCUSIÓN}

La riqueza de las especies consumidas (40 especies) por el venado cola blanca en Campeche, México fue similar a la reportada por DiMare (1994) para un bosque tropical caducifolio de Costa Rica. Sin embargo, en hábitats tropicales, la diversidad vegetal en la dieta del venado ha variado de 25 (Mendoza, 2003) hasta 125 especies (Granado, 1989), y ésta depende más de la estación y de la calidad del hábitat, que de la latitud y el tamaño de la especie (Arceo et al., 2005). Los valores mayores de la diversidad en la época de lluvia coincide con lo reportado por Granado (1989), pero difiere con DiMare (1994) y Arceo et al. (2005) quienes indican una mayor diversidad en la época seca. No obstante, el consumo de MS se relaciona con una mayor diversidad y disponibilidad de forraje verde, el cual es más común en la época de lluvia (Moen, 1978). Al respecto, Gallina, Maury \& Serrano (1981) encontraron una dieta más diversa en la época de lluvia, esto coincide con un mayor consumo de herbáceas, posiblemente por su suculencia, contenido nutricional o una mayor apetencia por estas (Clemente, 1984; Ramírez, 2004; López-Pérez, SerranoAzpeitia, Aguilar-Valdez \& Herrera-Corredor, 2012). En este estudio, la mayor diversidad de la dieta ocurrió en la época de lluvia y coincidió, de igual manera, con un mayor consumo de herbáceas, debido a su disponibilidad y mayor valor nutricional durante esa época (Hanley, 1982; Clemente, 1984; Gallina, Bello, Contreras \& Delfin-Alfonso 2010). Adicionalmente, y de acuerdo con Mautz (1976) y Moen (1978), en la época de mayor abundancia y calidad de forraje (como es la época de lluvia), el venado acumula reservas energéticas para utilizarlas 


\section{CUADRO 4}

Frecuencias e intervalos de confianza simultáneos de Bonferroni sobre el consumo de estratos vegetativos por el venado cola blanca en un campo experimental de Campeche, México

TABLE 4

Frequency and simultaneous confidence intervals of Bonferroni on the intake of vegetative strata by white tailed deer in an experimental unit of Campeche, México

\begin{tabular}{|c|c|c|c|c|c|c|c|}
\hline \multirow{3}{*}{$\begin{array}{c}\text { Estrato } \\
\text { vegetativo }\end{array}$} & \multicolumn{7}{|c|}{ Época de lluvia } \\
\hline & $\begin{array}{c}\text { Biomasa } \\
\text { disponible }\end{array}$ & $\begin{array}{c}\text { Frecuencia } \\
\text { esperada }\end{array}$ & $\begin{array}{l}\text { Frecuencia } \\
\text { observada }\end{array}$ & $\begin{array}{c}\text { Proporción } \\
\text { esperada }\end{array}$ & $\begin{array}{c}\text { Proporción } \\
\text { observada }\end{array}$ & $\begin{array}{c}\text { Intervalo } \\
\text { Bonferroni }\end{array}$ & \multirow[t]{2}{*}{ Uso } \\
\hline & $\mathrm{kgMS} / \mathrm{ha}$ & $\left(E i=n p_{i o}\right)$ & $\left(O_{i}\right)$ & $\left(p_{i o}\right)$ & $\left(p_{i}=O_{i} / n\right)$ & $(\mathrm{p}<0.05)$ & \\
\hline Pasto & 275 & 74 & 0 & 0.344 & 0 & $0 \leq \mathrm{p} \leq 0$ & $<$ \\
\hline Herbáceo & 214 & 57 & 113 & 0.268 & 0.528 & $0.443 \leq \mathrm{p} \leq 0.615$ & $>$ \\
\hline Arbustivo & 286.7 & 77 & 97 & 0.359 & 0.453 & $0.368 \leq p \leq 0.540$ & $>$ \\
\hline \multirow[t]{4}{*}{ Arbóreo } & 23.8 & 6 & 4 & 0.030 & 0.019 & $-0.004 \leq p \leq 0.042$ & $=$ \\
\hline & \multicolumn{7}{|c|}{$X^{2}=60$ g.l. $=3 \quad p<0.0001$} \\
\hline & \multicolumn{7}{|c|}{ Época de transición } \\
\hline & $\mathrm{kgMS} / \mathrm{ha}$ & $\left(E i=n p_{i o}\right)$ & $\left(O_{i}\right)$ & $\left(p_{i o}\right)$ & $\left(p_{i}=O_{i} / n\right)$ & $(\mathrm{p}<0.05)$ & \\
\hline Pasto & 60 & 50 & 13 & 0.195 & 0.050 & $0.016 \leq \mathrm{p} \leq 0.084$ & $<$ \\
\hline Herbáceo & 108 & 90 & 81 & 0.351 & 0.314 & $0.242 \leq \mathrm{p} \leq 0.386$ & $=$ \\
\hline Arbustivo & 129.5 & 109 & 152 & 0.421 & 0.589 & $0.513 \leq \mathrm{p} \leq 0.666$ & $>$ \\
\hline \multirow[t]{4}{*}{ Arbóreo } & 10.4 & 9 & 12 & 0.034 & 0.047 & $0.014 \leq \mathrm{p} \leq 0.079$ & $=$ \\
\hline & \multicolumn{7}{|c|}{$X^{2}=47$ g.l. $=3 p<0.0001$} \\
\hline & \multicolumn{7}{|c|}{ Época de seca } \\
\hline & $\mathrm{kgMS} / \mathrm{ha}$ & $\left(E i=n p_{i o}\right)$ & $\left(O_{i}\right)$ & $\left(p_{i o}\right)$ & $\left(p_{i}=O_{i} / n\right)$ & $(p<0.05)$ & \\
\hline Pasto & 27 & 18 & 18 & 0.142 & 0.142 & $0.064 \leq \mathrm{p} \leq 0.219$ & $=$ \\
\hline Herbáceo & 54.16 & 36 & 15 & 0.285 & 0.118 & $0.047 \leq \mathrm{p} \leq 0.190$ & $<$ \\
\hline Arbustivo & 99.5 & 66 & 85 & 0.523 & 0.669 & $0.565 \leq \mathrm{p} \leq 0.774$ & $>$ \\
\hline Arbóreo & 9.5 & 6 & 9 & 0.050 & 0.071 & $-0.594 \leq \mathrm{p} \leq 0.128$ & $=$ \\
\hline \multicolumn{8}{|c|}{$\mathrm{X}^{2}=19$ g.l. $=3 \mathrm{p}<0.0003$} \\
\hline
\end{tabular}

$>$ seleccionado; = usado de acuerdo a su disponibilidad; < evitado.

$>$ selected; $=$ used according to availability; $<$ avoided.

\section{CUADRO 5}

Capacidad de carga y carga animal para el venado cola blanca (venados/ha) en un campo experimental de Campeche, México

TABLE 5

Carrying capacity and stocking rate for White tailed deer (deer/ha) in an experimental unit of Campeche, Mexico

\begin{tabular}{lcccc}
\multicolumn{5}{c}{$\begin{array}{c}\text { Capacidad de carga } \\
\text { Estrato vegetativo }\end{array}$} \\
\multicolumn{1}{c}{ Pasto } & Herbáceo & Arbustivo & Arbóreo & 1.08 \\
\multicolumn{1}{c}{0.30} & 0.31 & 0.43 & 0.04 & \\
Estrato vegetativo & Lluvia & Transa animal & 0.07 \\
Pasto & 1.09 & 0.18 & 0.13 \\
Herbáceo & 0.85 & 0.32 & 0.25 \\
Arbustivo & 1.14 & 0.38 & 0.02 \\
Arbóreo & 0.09 & 0.03 & 0.47 \\
Total & 3.18 & 0.92 &
\end{tabular}


en el periodo de mayor estrés, que en climas cálidos, se da en la época seca (López-Pérez et al. 2012); en esta época, la acumulación de reservas permite compensar la baja calidad del forraje para disminuir el riesgo de enfermedades y una baja tasa de parición (Smith, Holder, Hayes, \& Silver, 1975; Clemente, 1984; Arceo et al., 2005 ). En contraste López-Pérez et al. (2012) encontraron una dieta más digestible en el otoño en el estado de Morelos y en la Mixteca Poblana, los arbustos fueron los más consumidos y los que aportaron mayor cantidad de MS (Villarreal-Espino et al., 2011).

Tres de las 15 familias botánicas, Fabaceae, Malvaceae y Convolvulaceae fueron las más frecuentes en la dieta del venado cola blanca en el área de estudio. $\mathrm{Al}$ respecto, Clemente (1984) reportó que las especies de las familias Fabaceae y Convolvulaceae fueron también las más frecuentes en la dieta del venado. Asimismo, identificó que dichas especies contenían los niveles más altos de proteína, calcio y fósforo, y una digestibilidad de MS mayor al $50 \%$. Por su parte, López-Pérez et al. (2012), reportó que la especie más frecuente en la dieta del venado en el estado de Morelos pertenece a la familia Malvaceae, que presentó las mejores características nutricionales (proteína cruda, digestibilidad) en la dieta. Adicionalmente, se menciona que el consumo de especies vegetales por el venado está en función de su valor nutritivo como lo especifica Moore \& Johnson (1967).

En este estudio, las especies más comunes en la dieta del venado cola blanca fueron: Bauhinia divaricata (lluvia, transición y seca), Eugenia sp. (lluvia y transición) y Leucaena leucocephala (seca). La frecuencia alta de $B$. divaricata puede estar relacionada con su alto potencial forrajero (Sosa, Pérez, Ortega \& Zapata, 2004), al respecto, Zapata, Bautista \& Aster (2009) determinaron que la época seca fue la de mejor calidad forrajera, ya que presentó la mejor respuesta al corte, mayor número de rebrotes, los mejores rendimientos, alta digestibilidad $(>40 \%)$ y un $14 \%$ de proteína cruda (Sosa et al., 2004).
En un estudio realizado en Cuba sobre las preferencias de ramoneo del venado cola blanca, Hernández, Aranda \& Uvalle (2001) reportan que Eugenia buxifolia fue la especie preferida y Weber (2005) reporta la preferencia de Eugenia sp. en la reserva de Calakmul, Campeche, México. Por su parte, Plata et al. (2009) encontraron que L. leucocephala fue la especie más consumida en la dieta de venados en cautiverio en la península de Yucatán.

La mayor frecuencia de herbáceas en la dieta del venado cola blanca en la época de lluvia, coincide con diversos estudios realizados en hábitats tropicales, templados y áridos (Kie, Drawe \& Scott, 1980; Gallina et al., 1981; Granado, 1989; DiMare, 1994; Arceo et al., 2005; Vásquez, 2009); de igual forma, la dominancia de las arbustivas en la temporada seca coincidió con Gallina (1977) y Clemente (1984). La asociación registrada entre la temporada seca con los pastos, tuvo relación con la baja disponibilidad de biomasa de arbustivas y arbóreas.

Diversos estudios reportan que el venado cola blanca selecciona especies arbustivas (Galindo-Leal \& Weber, 1998) e indican que estas especies, además de alimento, le proporcionan al venado cobertura (Gallina et al., 2010; Aguilera-Reyes et al., 2013), y que a diferencia de las herbáceas, las arbustivas mantienen una producción de biomasa relativamente constante a través del año. Al respecto, Clemente (1984) reporta que el cambio en la composición nutricional de las arbustivas es menor al de las plantas en los otros estratos vegetativos, lo que explica su alta frecuencia en la dieta durante la época seca. Por lo tanto, la menor preferencia de los pastos, en este estudio, pudo deberse a su baja digestibilidad comparada con la de las herbáceas y arbustivas (Galindo-Leal \& Weber, 1998; Dyer, Goldberg, Turkington \& Sayre, 2001).

Ramírez (2004) clasifica al venado cola blanca como un herbívoro que selecciona forrajes de alta calidad para cubrir su tasa metabólica y demanda alta de energía; en el proceso de forrajeo, busca alimentos suculentos y nutritivos y consume menos celulosa que las otras especies con las que coexiste (Duncan, 
Ginane, Gordon \& Orskov, 2003; Clemente et al., 2005).

El índice alto de equitatividad durante el año reflejó la uniformidad de la abundancia relativa de las especies durante el estudio, esta uniformidad fue similar a la obtenida por González \& Briones-Salas (2012). El índice de similitud entre las épocas del año se relacionó con la producción de forraje y su estado fenológico, existiendo mayor similitud entre la época de lluvia y transición, esto posiblemente debido a que la calidad del forraje en ambas épocas fue similar. En contraste, en la época seca disminuyó la diversidad de especies en la dieta, efecto que ocasionó una disimilitud entre ésta y las demás épocas del año.

Los valores de $K$ obtenidos en este estudio se encuentran dentro del intervalo reportado en diversos estudios en México (Mandujano, 2007; Plata, Mendoza, Viccon, Bárcena \& Clemente, 2011, Villareal-Espino et al., 2011). La K más alta se estimó con la biomasa total y la CA mayor ocurrió en la época de lluvia, cuando las condiciones climáticas favorecen el crecimiento y la diversidad de plantas (Valles, Castillo \& Hernández, 1992; Sosa, Cabrera, Pérez \& Ortega, 2008). Al respecto, Mandujano (2008) determinó que en las regiones tropicales, a mayor precipitación, la $\mathrm{K}$ y la densidad de venados se incrementan. Por su parte, Contreras (2008) en un bosque tropical húmedo de Tabasco, determinó una densidad de 0.01 venados/ha de $O$. v. thomasi, la cual se aproxima a la $\mathrm{K}$ calculada en este estudio, considerando solo el forraje limitante (biomasa proveniente de las especies arbóreas) como lo propone Holechek et al. (1995). La CA en la época restrictiva (seca) fue mayor $(0.47$ venados/ha) a la reportada para bosques tropicales de Jalisco (0.152 venados/ha) y Chiapas (0.099 venados/ha) (Naranjo, Guerra, Bodmer \& Bolaños, 2004; Mandujano, 2008).

La dieta del venado cola blanca en la región costera de Campeche varió con la época del año y consistió principalmente de especies arbustivas. Dos especies, Eugenia sp. y
Bauhinia divaricata fueron las especies más consumidas, su presencia podría ser indicador de una buena calidad del hábitat o su ausencia indicaría la necesidad de realizar mejoras del hábitat en el área de distribución de $O$. v. thomasi. La $\mathrm{K}$ más cercana a la densidad poblacional de la subespecie fue con la MS de las arbóreas; sin embargo, para manejar adecuadamente al venado cola blanca es necesario considerar también los requerimientos de las especies con las que el venado comparte su hábitat en Campeche, México. La CA más alta ocurrió en la época de lluvia y la más baja en la seca. Este representa un estudio pionero sobre Odocoileus virginianus thomasi, pero se requieren estudios complementarios para detallar la composición de la dieta, la disponibilidad y uso del hábitat en todo su ámbito de distribución, en donde se consideren además aspectos nutricionales y sanitarios.

\section{RESUMEN}

En Campeche, México no existen estudios de la dieta de Odocoileus virginianus thomasi; a pesar de la existencia de 122 Unidades de Manejo para la Conservación de la Vida Silvestre (UMAS) en donde se realiza aprovechamiento del venado cola blanca. Los objetivos del trabajo fueron conocer la composición botánica de la dieta y su cambio estacional, la disponibilidad de forraje, la capacidad de carga y carga animal de $O$. virginianus thomasi. El trabajo se realizó de octubre 2010 a mayo 2012 en el Campo Experimental del Colegio de Postgraduados en Campeche, México. La composición de la dieta se determinó mediante el análisis de heces de venado y material de referencia con la técnica microhistológica; la disponibilidad de forraje se estimó con el método de Adelaide; la capacidad de carga utilizando la presión de pastoreo y la carga animal considerando el forraje disponible y $35 \%$ de eficiencia de utilización. La dieta incluyó 40 especies de 15 familias. La riqueza de especies más alta fue de 29 especies en la época de lluvia; las arbustivas fueron preferidas en las tres épocas del año y las herbáceas en la época de lluvia. La composición de la dieta, disponibilidad de forraje, la capacidad de carga y carga animal variaron a través del año. La capacidad de carga fluctuó entre 0.04 a 1.08 venados/ha.

Palabras clave: capacidad de carga, carga animal, variación estacional, hábitat tropical, hábitos alimenticios, análisis microhistológico. 


\section{REFERENCIAS}

Aguilera-Reyes, U., Sánchez-Cordero, V., Ramírez-Pulido, J., Monroy-Vilchis, O., García, L. G., \& Janczur, M. (2013). Hábitos alimentarios del venado cola blanca Odocoileus virginianus (Artiodactyla: Cervidae) en el Parque Natural Sierra Nanchititla, Estado de México. Revista de Biología Tropical, 61(1), 243-253.

Akçakaya, H. R., Burgman, M., \& Ginzburg, L. (1999). Applied Population Ecology: principles and computer exercises using RAMAS EcoLab 2.0 (2nd ed., p. 285). Sunderland, MA: Sinauer.

Allison, C. D. (1985). Factors affecting forage intake by range ruminants: a review. Journal of Range Management, 38, 305-311.

Andrew, M. H., Noble, I. R., \& Lange, R. T. (1979). A non-destructive method for estimating the weights of forage on shrubs. Australian Rangeland Journal, l(3), 225-231.

Anthony, R. G., \& Smith, N. S. (1974). Comparison of rumen and fecal analysis to describe deer diets. Journal of Wildlife Management, 38(3), 535-540.

Arceo, G. (2003). Hábitos alimentarios del venado cola blanca (Odocoileus virginianus) en el bosque tropical caducifolio de Chamela, Jalisco (Tesis de Maestría). Universidad Nacional Autónoma de México, México, D.F.

Arceo, G., Mandujano, S., \& Gallina, S. (2005). Diet diversity of white-tailed deer (Odocoileus virginianus) in a tropical dry forest in Mexico. Mammalia, 69, 159-168.

Bailey, D. W., Groos, J. E., Laca, E. A., Rittenhouse, L. R., Coughenour, M. E., Swift, D., \& Sims, P. L. (1996). Mechanism that result in large herbivore grazing distribution patterns. Journal of Range Management, 49(5), 386-400.

Byers, C. R., Steinhorst, R. K., \& Krausman, P. R. (1984). Clarification of a technique for analysis of utilizationavailability data. Journal of Wildlife Management, 48(3), 1050-1052.

Clemente, S. F. (1984). Utilización de la vegetación nativa del venado cola blanca (Odocoileus virginianus). (Tesis inédita de Maestría). Colegio de Postgraduados, Montecillo, Estado de México, México.

Clemente, S. F., Riquelme, E., Mendoza, G. D., Bárcena, R., González, S., \& Ricalde, R. (2005). Digestibility of forage diets of white-tailed deer (Odocoileus virginianus, Hays) using different ruminal fluid inocula. Journal of Applied Animal Research, 27(3), 71-76.

Contreras, F. M. (2008). Ecología poblacional del venado cola blanca (Odocoileus virginianus thomasi) en la R/A San Joaquín municipio de Balancán, Tabasco, México (Tesis inédita de licenciatura). Universidad Juárez Autónoma de Tabasco, México.
Cordova, F. J., Wallace, J. D., \& Pieper, R. D. (1978). Forage intake by grazing livestock; a review. Journal of Range Management, 31(6), 430-436.

Cuanalo, C. E., Ojeda, E., Santos, A., \& Ortiz, C. A. (1989). Provincias, regiones y subregiones terrestres de México. Chapingo, México: Colegio de Postgraduados, Centro de Edafología, México.

DiMare, M. I. (1994). Hábitos alimentarios del venado cola blanca en la Isla San Lucas, Puntarenas, Costa Rica (pp. 73-90). In C. Vaughan, \& M. Rodríguez (eds.). Ecología y manejo del venado cola blanca en México y Costa Rica. Heredia, Costa Rica: EUNA.

Distel, R. A., Villalba, J. J., \& Laborde, H. E. (1994). Effects of early experience on voluntary intake of low-quality roughage by sheep. Journal of Animal Science, 72(5), 1191-1195.

Duncan, A. J., Ginane, C., Gordon, I. J., \& Orskov, E. R. (2003). Why do herbivores select mixed diets? In L. Mannetje, A. L. Ramírez, C. A. A. Sandoval \& V. F. C. $\mathrm{Ku}$ (Eds.), Matching herbivore nutrition to ecosystems biodiversity (pp. 195-212). VI International Symposium on the nutrition of herbivores. Yucatán, México.

Dyer, A. R., Goldberg, D. E., Turkington, R., \& Sayre, C. (2001). Effects of growing conditions and source habitat on plant traits and functional group definition. Functional Ecology, 15(1), 85-95.

Enríquez, Q. J., Bolaños, A. E., \& Meléndez, N. F. (1999). Tecnología para la producción y manejo de forrajes tropicales en México (Libro Técnico Núm. 7). Papaloapan, Veracruz: INIFAP.

Forbes, T. D. A. (1988). Researching the plant-animal interface: The investigation of ingestive behavior in grazing animals. Journal of Animal Science, 66(9), 2369-2379.

Fracker, S. B., \& Brichle, J. A. (1944). Measuring the local distribution of Rikes. Ecology, 25: 283-303.

Galindo-Leal, C., \& Weber, M. (1998). El Venado de la Sierra Madre Occidental: Ecología, Conservación y Manejo. México: Edicusa-Conabio.

Gallina, S. (1977). Hábitos alimenticios del venado cola blanca (Odocoileus virginianus Rafinesque) en la Reserva de La Michilia, estado de Durango. (Tesis inédita de licenciatura). Universidad Nacional Autónoma de México, México, D. F.

Gallina, S., Maury, E., \& Serrano, V. (1981). Foods habits of white-tailed deer. In P. F. Efolliott, \& S. Gallina (Ed.), Deer Biology, Habitat Requirements and Management in Western North America (pp. 135148). Mexico, D.F.: Instituto de Ecología A.C.

Gallina, S. (1993). White-tailed deer and cattle diets at La Michilia, Durango, Mexico. Journal of Range Management, 46(6), 487-492. 
Gallina, S., Bello, J., Contreras, C., \& Delfin-Alfonso, C. (2010). Daytime bedsite selection by the Texan white-tailed deer in xerophyllous brushland, Northeastern, Mexico. Journal of Arid Environments, 74(3), 373-377.

García, E. (1981). Modificaciones al sistema de clasificación de Köppen para adaptarlo a las condiciones de la República Mexicana. México: Instituto de Geografía, Universidad Nacional Autónoma de México.

Gonzáles, G., \& Briones, S. M. (2012). Dieta de Odocoileus virginianus (Artiodactyla: Cervidae) en un bosque templado del norte de Oaxaca, México. Revista de Biología Tropical, 60 (1), 447-457.

Gotelli, N. J. (2008). Logistic population growth. In N. J. Gotelli (Ed.), A primer of ecology (pp. 25-48). Ma, USA: Sinauer Associates Inc. Sunderland.

Granado, A. (1989). Dieta del venado caramerudo (Odocoileus virginianus gymnotis) en El Socorro, estado Guarico. (BSc. Tesis inédita). Universidad Central de Venezuela, Caracas.

Halls, L. K. (1984). White tailed deer: ecology and management. A Wildlife Management Institute Book. U. S. A.: Stackpole Books.

Hanley, T. A. (1982). The nutritional basis for food selection by ungulates. Journal of Range Management, 35(2), 146-151.

Hernández, M. F., Aranda, R. R., \& Uvalle, J. L. (2001). Observaciones sobre el ramoneo del venado cola blanca (Odocoileus virginianus) en un bosque semidesiduo en la provincias del Pinar del Rio y Matanzas, Cuba. Crónica Forestal y del Ambiente, 16, 67-73.

Holechek, J. L. (1982). Sample preparation technique for microhistological analysis. Journal of Range Management, 35(2), 267-268.

Holechek, J. L., Pieper, R. D., \& Herbel, C. H. (1995). Range management principles and practices (2nd ed., pp. 177-214). Englewood Cliffs, NJ.: Prentice Hall.

Holechek, J. L., Galt, D., Joseph, J., Navarro, J., Kumalo, G., Molinar, F., \& Thomas, M. (2003). Moderate and light cattle grazing effects on Chihuahuan Desert rangelands. Journal of Range Management, 56(2), 133-139.

Hubálek, Z. (1982). Coefficients of association and similarity, based on binary (presence and absence) data: an evaluation. Biological Reviews, 57(4), 669-689.

Janis, C. M., Damuth, J., \& Theodor, J. M. (2000). Miocene ungulates and terrestrial primary productivity: Where have all the browsers gone? Proceedings of the National Academy of Sciences, 97(14), 7899-7904.

Kie, J. G., Drawe, D. L., \& Scott, G. (1980). Changes in diet and nutrition with increased herd size in Texas white-tailed deer. Journal of Range Management, 33(1), 28-34.
Lemos, E. J. A., Rojas, G. R., \& Zúñiga, V. J. (2005). Técnicas para el estudio de poblaciones de fauna silvestre. México. Comisión Nacional para el Conocimiento y Uso de la Biodiversidad y Universidad Nacional Autónoma de México.

López-Pérez, E., Serrano-Aspeitia, N., Aguilar-Valdés, B., \& Herrera-Corredor, A. (2012). Composición nutricional de la dieta del venado cola blanca (Odocoileous virginianus sp. mexicanus) en Pitzotlán, Morelos. Revista Chapingo Serie Ciencias Forestales $y$ del Ambiente, 18(2), 219-229.

Mandujano, S. (2004). Análisis bibliográfico de los estudios de venado en México. Acta Zoológica Mexicana, 20(1), 211-251.

Mandujano, S. (2007). Carrying capacity and potential production of ungulates for human use in a Mexican tropical dry forest. Biotropica, 39(4), 519-524.

Mandujano, S. (2008). Precipitación, capacidad de carga y potencial de uso de ungulados en un bosque tropical seco del Pacífico Mexicano (pp. 637-660, Vol. II). In C. Lorenzo, E. Espinoza-Medinilla, \& J. Ortega (Ed.), Avances en el Estudio de los Mamíferos de México. México: AMMAC.

Martínez, A., Molina, V. M., González, F., Marroquín, J. S., \& Navar, J. (1997). Observations of white-tailed deer and cattle diets in Mexico. Journal of Range Management, 50(3), 253-257.

Mautz, W. W., Silver, H., Holter, J. B., Hayes, H. H., \& Urban W. E. (1976). Digestibility and related nutritional data for seven northern deer browse species. Journal of Wildlife Management, 40(4), 630-683.

Mendoza, Ch. (2003). Alimentación del venado cola blanca. Manejo de venado cola blanca en la UNEXMIR (pp. 1-8). Yucatán, México: Universidad Marista, Mérida.

Moen, A. (1978).Seasonal changes in heart rates, activity, metabolism, and forage intake of white-tailed deer. Journal of Wildlife Management, 42(4), 715-738.

Moore, H. W., \& Johson, M. F. (1967). Nature of Deer Browsing on Hardwood Seedlings and Sprouts. Journal of Wildlife Management, 31(2), 351-353.

Naranjo, E. J., Guerra, M. M., Bodmer, R. E., \& Bolaños, J. E. (2004). Subsistence Hunting by Three Ethnic Groups of the Lacandon Forest, Mexico. Journal of Ethnobiology, 24(2), 233-253.

Neu, C. W., Byers, C. R., \& Peek, J. M. (1974). A technique for analysis of utilization-availability data. Journal of Wildlife Management, 38(3), 541-545.

National Research Council. (1987). Predicting feed intake of food-producing animals. Washington, D.C: National Academy Press.

Paladines, O., \& Lascano, C. E. (1983). Recomendaciones para evaluar germoplasma bajo pastoreo. In C. E. Lascano (Ed.), Germoplasma forrajero bajo pastoreo 
en pequeñas parcelas, (Vol. 1., pp. 165-183). Colombia: CIAT.

Peña, J. M., \& Habib, R. (1980). La Técnica Microhistológica; un método para determinar la composición botánica de la dieta de herbivoros (Serie Técnico Científica, Vol. 1, $\mathrm{N}^{\mathrm{o}} 6$, p. 82). México: Instituto Nacional de Investigaciones Pecuarias. Secretaría de Agricultura y Recursos Hidráulicos.

Plata, F. X., Ebergeny, S., Reséndiz, J. L., Villareal, O., Bárcena, R., Viccon, J. A., \& Mendoza, G. D. (2009). Palatabilidad y composición química de alimentos consumidos en cautiverio por el venado cola blanca de Yucatán (Odocoileus virginianus yucatanensis). Archivos de Medicina Veterinaria, 41(2), 123-129.

Plata, F. X., Mendoza, G. D., Viccon, J. A., Bárcena, R., \& Clemente, C. 2011. Comparación de métodos basados en los requerimientos nutricionales y disponibilidad de biomasa para estimar la capacidad de carga para venado cola blanca. Archivos de Medicina Veterinaria, 43(1), 41-50.

Provenza, F. (1995). Postingestive feedback as an elementary determinant of food preference and intake in ruminants. Journal of Range Management, 48(1), 2-17.

Ramírez, G. R. (2004). Nutrición del venado cola blanca (pp.138-142). México: Universidad Autónoma de Nuevo León.

Robbins, T. C. (2001). Wildlife feeding and nutrition (2nd ed., p. 352). San Diego, New York, Boston: Academic Press.

SAS Institute. 2008. User's Guide: Statistics, version 9.2. Cary, Carolina del Norte, EE.UU.: Statistical Analysis System, Institute.

SEMARNAT. (2011). Compendio de estadísticas ambientales 2010. (Recuperado de: http://aplicaciones.semarnat.gob.mx/estadisticas/compendio2010/10.100.13.5 8080/ibi apps/WFServlet85d9. html en 07/02/11).

Smith, S. H., Holder, J. B., Hayes, H. H., \& Silver, H. (1975). Protein requirements of white tailed deer fawns. Journal of Wildlife Management, 39, 582-589.
Sosa, R. E., Pérez, R. D., Ortega, R. L., \& Zapata, B. G. (2004). Evaluación del potencial forrajero de árboles y arbustos tropicales para la alimentación de ovinos. Técnica Pecuaria en México, 42(2), 129-144.

Sosa, R. E., Cabrera, T. E., Pérez, R. D., \& Ortega, R. L. (2008). Producción estacional de materia seca de gramíneas y leguminosas forrajeras con cortes en el estado de Quintana Roo. Técnica Pecuaria en México, 46(4), 413-426.

Stuth, J. W., \& Sheffield, W. J. (2001). Determining carrying capacity for combinations of livestock, white-tailed deer and exotic ungulates. In Wildlife Management Handbook (pp. 5-12), Texas, USA: Texas A\&M University System.

Valles, B., Castillo, E., \& Hernández, T. (1992). Producción estacional de leguminosas forrajeras en Veracruz, México. Pasturas Tropicales, 14, 32-36.

Vásquez, F. Y. (2009). Composición botánica de la dieta de venado cola blanca (Odocoileous virginianus subespecie mexicanus) en Pitzotlán, Morelos (Tesis inédita de licenciatura). Universidad Autónoma Chapingo, Texcoco, Edo. de México, México.

Villarreal-Espino, O. A., Plata-Pérez, F. X., CamachoRonquillo, J. C., Hernández-Hernández, J. E., Franco-Guerra, F. J., Aguilar-Ortega, B., \& MendozaMartínez, G. D. (2011). El venado cola blanca en la mixteca poblana. Therya, 2(2), 103-110.

Weber, M., García M. G., \& Reyna-Hurtado, R. (2006). The Tragedy of the Commons: Wildlife Management Units in Southeastern Mexico. Wildlife Society Bulletin, 34(5), 1480-1488.

Weber, M. (2005). Ecology and conservation of sympatric tropical deer populations in the Greater Calakmul Region, Mexico (Tesis doctoral). University of Durham.

Zapata, B. G., Bautista, Z. F., \& Aster, C. M. (2009). Caracterización forrajera de un sistema silvopastoril de vegetación secundaria con base en la aptitud del suelo. Técnica Pecuaria en México, 47(3), 257-270.

Zar, L. H. (1999). Bioestatistical Analysis. Nueva Jersey, EE.UU: Prentice Hall, Upper Saddle River. 